\title{
On characterization of the hypercenters of fuzzy groups
}

\author{
K. O. Grin \\ Oles Honchar Dnipropetrovsk National University \\ Dnipropetrovsk, 49050.E-mail: catherine.grin@gmail.com
}

Ми продовжили вивчення властивостей верхнього центрального ряду довільної фаззі-групи. Точніше кажучи, було отримано досить явний опис членів верхнього центрального ряду.

Ключові слова: фаззі-група, верхній центральний ряд, другий гіперцентр, верхній гіперцентр.

Мы продолжили изучение свойств верхнего центрального ряда произвольной фаззи-группы. Точнее говоря, было получено достаточно явное описание членов верхнего центрального ряда.

Ключевые слова: фаззи-группа, верхний центральный ряд, второй гиперцентр, верхний гиперцентр.

We continue the study of the properties of the upper central series for arbitrary fuzzy group. More precisely, a quite explicit description of the members of the upper central series has been obtained.

Key words: fuzzy group, upper central series, second hypercenter, upper hypercenter.

Let $G$ be a group with a multiplicative binary operation denoted by juxtaposition and identity $e$. We recall that a fuzzy subset $\gamma: G \rightarrow[0,1]$ is said to be a fuzzy group on $\boldsymbol{G}$ (see, for example, [[5], S. 1.2]), if it satisfies the following conditions:

$$
\begin{aligned}
& (\text { FSG 1) } \gamma(x y) \geq \gamma(x) \wedge \gamma(y) \text { for all } x, y \in G, \\
& \left(\text { FSG 2) } \gamma\left(x^{-1}\right) \geq \gamma(x) \text { for every } x \in G .\right.
\end{aligned}
$$

Here and everywhere we adopt the usual convention on the operator wedge $\wedge$ (and on the operator vee $\vee$ ). If $W$ is a subset of $[0,1]$, then denote by $\wedge W$ the greatest lower bound of $W$ and denote by $\vee W$ the least upper bound of $W$. If $W=\{a, b\}$, then, as usual, instead of $\wedge W$ we will write $a \wedge b$, and instead of $\vee W$ we will write $a \vee b$. We assume that the least upper bound of the empty set is 0 , and the greatest lower bound of the empty set is 1 .

However we remark that we deliberately replace the standard expression a fuzzy subgroup of $G$ by a fuzzy group on $G$ in order to avoid possible misunderstanding in the sequel and to emphasize that a fuzzy group is in fact a function defined on a group $G$. For example, if $\gamma, \kappa$ are the fuzzy group on $G$ and $\gamma \subseteq \kappa$, occurs, we will say that $\gamma$ is a fuzzy subgroup of $\kappa$ and denote this by $\gamma \preccurlyeq \kappa$. If $\gamma$ is a fuzzy subgroup of $\kappa$, then $\gamma(e) \leq \kappa(e)$. Fuzzy subgroup $\gamma$ of $\kappa$ is called unitary, if $\gamma(e)=\kappa(e)$. If $\gamma$ is an 
arbitrary fuzzy subgroup of $\kappa$, then clearly $\gamma^{*}=\gamma \cup \chi(e, \kappa(e))$ is a fuzzy subgroup of $\kappa$. Moreover, $\gamma^{*}(x)=\gamma(x)$ for all $x \neq e$ and $\gamma^{*}(x)=\kappa(e)$.

Fuzzy group theory, as well as other fuzzy algebraic structures, was introduced very soon after the beginning of fuzzy set theory. Many basic results of the theory were collected in the book [5]. From our point of view, these results are not always systematized, and the methodology and the research tools seem to be at an initial stage. The obtained results apply to the different fields but they are characterized by initial almost everywhere. The development of complete theory of fuzzy groups has not taken place yet. The first natural task here appears to be the description of all fuzzy subgroups of a given fuzzy group, defined on $G$. The second main task is the investigation of the structure of a fuzzy group on $G$ based on its algebraic properties. One of the important concept not only in group theory but also in the whole algebra is the concept of nilpotency. It was introduced for fuzzy groups too ( see [5], Chapters 3.2, and the papers [1], [2], [3]). This definition was given with use of the lower central series. Meanwhile there are other definitions in abstract group theory. One of them, not less important, is based on the concept of upper central series. In fuzzy group theory the upper central series haven't constructed before. The constructing of the upper central series in arbitrary fuzzy group $\gamma$ was carried out only in the recent paper [4]. In this paper we continue the study of the properties of the upper central series for arbitrary fuzzy group defined on a group $G$. More precisely, a quite explicit description of the members of the upper central series is given in the paper.

Recall the following definition. If $X$ is a set, for every subset $Y$ of $X$ and every $a \in[0,1]$ we define a fuzzy subset $\chi(Y, a)$ as follows:

$$
\chi(Y, a)= \begin{cases}a, & \text { if } x \in Y ; \\ 0, & \text { if } x \notin Y .\end{cases}
$$

Clearly $\chi(H, a)$ is a fuzzy group on $G$ for every subgroup $H$ of $G$. If $Y=\{y\}$, then instead of $\chi(\{y\}, a)$ we will write shorter $\chi(y, a)$. A fuzzy subset $\chi(y, a)$ is called a fuzzy point (or fuzzy singleton).

Let $L$ be a subgroup of $G$ and $\gamma$ be a fuzzy subgroup on $G$. Denote the function $\left.L\right|^{\gamma}$ by the following rule:

$$
\left.L\right|^{\gamma}(x)= \begin{cases}\gamma(x), & \text { if } x \in L ; \\ 0, & \text { if } x \notin L .\end{cases}
$$

Let $G$ be a group, $\gamma$ be a fuzzy subgroup on $G$. Then the center $\mathfrak{z}(\gamma)$ of $\gamma$ is an union of all fuzzy points $\chi(z, a) \subseteq \gamma$ such that $\chi(z, a) \odot \chi(y, \gamma(y))=\chi(y, \gamma(y)) \odot \chi(z, a)$ for every $\chi(y, \gamma(y)) \subseteq \gamma$.

A fuzzy group $\gamma$ is called abelian, if $\mathfrak{z}(\gamma)=\gamma$.

We observed that in the theory of fuzzy groups the term "abelian" is used in different senses. We will use it in the traditional aspect.

Lemma 1. Let $G$ be a group, $\gamma$ be a fuzzy subgroup on $G$. Then the fuzzy points $\chi(x, a)$ and $\chi(y, b)$ are permutes if and only if $x y=y x$. 
Proof. Suppose that $\chi(x, a) \odot \chi(y, b)=\chi(y, b) \odot \chi(x, a)$. By Proposition 1 of paper [4] we have

$$
\chi(x, a) \odot \chi(y, b)=\chi(x y, a \wedge b) \text { and } \chi(y, b) \odot \chi(x, a)=\chi(y x, b \wedge a),
$$

so we obtain $x y=y x$.

Conversely, assume that $x y=y x$. Then

$$
\chi(x, a) \odot \chi(y, b)=\chi(x y, a \wedge b)=\chi(y x, b \wedge a)=\chi(y, b) \odot \chi(x, a) .
$$

Corollary 1. Let $G$ be a group, $\gamma$ be a fuzzy subgroup on $G$. Then $\mathfrak{z}(\gamma)$ is an union of all fuzzy points $\chi(z, a)$ such that $z \in \zeta(G)$. In other words, $\mathfrak{z}(\gamma)=\left.\zeta(\operatorname{Supp}(\gamma))\right|^{\gamma}$.

Proof. Suppose that $\chi(z, a) \in \mathfrak{z}(\gamma)$. Then $\chi(z, a) \odot \chi(y, \gamma(y))=\chi(y, \gamma(y)) \odot \chi(z, a)$ for every element $y \in \operatorname{Supp}(\gamma)$. By Lemma 1 it follows that $z y=y z$, since it is valid for every element $y \in \operatorname{Supp}(\gamma), z \in \zeta(\operatorname{Supp}(\gamma))$.

Conversely, assume that $z \in \zeta(\operatorname{Supp}(\gamma))$ and $a \leq \gamma(z)$. Then $z y=y z$ for each element $y \in \operatorname{Supp}(\gamma)$. Using again Lemma 1 we obtain that

$$
\chi(z, a) \odot \chi(y, \gamma(y))=\chi(y, \gamma(y)) \odot \chi(z, a),
$$

which follows that $\chi(z, a) \in \mathfrak{z}(\gamma)$.

Put $\zeta^{*}=\left.\zeta(\operatorname{Supp}(\gamma))\right|^{\gamma}$. Then $\zeta^{*}=\bigcup_{z \in \zeta(\operatorname{Supp}(\gamma))} \chi(z, \gamma(z))$. By above proved, $\zeta^{*} \subseteq$ $\zeta(\gamma)$. Conversely, let $\chi(z, a) \subseteq \zeta(\gamma)$. By above proved, $z \in \zeta(\operatorname{Supp}(\gamma))$. Since $\chi(z, a) \subseteq$ $\gamma, a \leq \gamma(z)$. It follows that $\chi(z, a) \subseteq \chi(z, \gamma(z)) \subseteq \zeta^{*}$.

Corollary 2. Let $G$ be a group, $\gamma$ be a fuzzy subgroup on $G$. Then $\gamma$ is abelian if and only if $\operatorname{Supp}(\gamma)$ is abelian.

Let $G$ be a group, $x, y \in G, a, b \in[0,1]$. Then a product $\chi\left(x^{-1}, a\right) \odot \chi\left(y^{-1}, b\right) \odot$ $\chi(x, a) \odot \chi(y, b)$ is called a commutator of $\chi(x, a)$ and $\chi(y, b)$ and will denoted by $[\chi(x, a), \chi(y, b)]$.

Let $G$ be a group, $\gamma, \eta$ be the fuzzy subgroups of $G$. Then a fuzzy commutator subgroup $[\gamma, \eta]$ is a fuzzy subgroup generated by all commutators $[\chi(x, \gamma(x)), \chi(y, \eta(y))]$ where $x \in \operatorname{Supp}(\gamma), y \in \operatorname{Supp}(\eta)$.

Corollary 3. Let $G$ be a group, $\gamma$ be a fuzzy subgroup on $G$. Then $\mathfrak{z}(\gamma)$ is an union of all fuzzy points $\chi(z, \gamma(z))$ such that $[\chi(z, \gamma(z)), \chi(y, b)] \subseteq \chi(e, \gamma(e))$.

Proof. Indeed, by Corollary 3 of Lemma 2 of paper [4] an inclusion $[\chi(z, \gamma(z)), \chi(y, b)] \subseteq \chi(e, \gamma(e))$ is equivalent to $\chi(z, \gamma(z)) \odot \chi(y, b)=\chi(y, b) \odot$ $\chi(z, \gamma(z))$. Now we can apply Corollary 1 .

We now are developing a criterion of being a fuzzy subgroup needed in the sequel. 
Lemma 2. Let $G$ be a group and $\gamma$ be a fuzzy subgroup on $G$. If $\lambda, \kappa \subseteq \gamma$, then $\lambda \odot \kappa \subseteq \gamma$, in particular, $\gamma \odot \gamma \subseteq \gamma$.

Proof. Let $x$ be an arbitrary element of $G$. We have

$$
(\lambda \odot \kappa)(x)=\bigvee_{y, z \in G, y z=x}(\lambda(y) \wedge \kappa(z))
$$

The inclusions $\lambda, \kappa \subseteq \gamma$ imply $\lambda(y) \wedge \kappa(z) \leq \gamma(y) \wedge \gamma(z)$. Since $\gamma$ is a fuzzy subgroup, $\gamma(y) \wedge \gamma(z) \leq \gamma(y z)$, thus

$$
(\lambda \odot \kappa)(x)=\bigvee_{y, z \in G, y z=x}(\lambda(y) \wedge \kappa(z)) \leq \bigvee_{y, z \in G, y z=x} \gamma(y z)=\gamma(x)
$$

Proposition 1. Let $G$ be a group and $\gamma$ be a fuzzy subset of $G$. Then $\gamma$ is a fuzzy subgroup if and only if the following assertion holds:

$($ FSG 3) $\chi(x, a), \chi(y, b) \subseteq \gamma$ imply $\chi(x, a) \odot \chi(y, b) \subseteq \gamma$ for all $x, y \in \operatorname{Supp}(\gamma)$, $\left(\right.$ FSG 4) $\chi(x, a) \subseteq \gamma$ implies $\chi\left(x^{-1}, a\right) \subseteq \gamma$ for every $x \in \operatorname{Supp}(\gamma)$.

Proof. Suppose first that $\gamma$ is a fuzzy group on $G$. Since $\gamma$ includes the functions $\chi(x, a)$ and $\chi(y, b)$ for every elements $x, y \in \operatorname{Supp}(\gamma)$, using Lemma 2, we obtain that $\chi(x, a) \odot \chi(y, b) \subseteq \gamma$.

Let $x$ be an arbitrary element of $\operatorname{Supp}(\gamma)$. Since $\chi(x, a) \subseteq \gamma, a \leq \gamma(x)$. We have $\left(\chi\left(x^{-1}, \gamma(x)\right)\right)\left(x^{-1}\right)=\gamma(x)$. Since $\gamma$ is a fuzzy group, $\gamma(x) \leq \gamma\left(x^{-1}\right)$. We note that if $y \neq x^{-1}$, then $\left(\chi\left(x^{-1}, \gamma(x)\right)\right)(y)=0$, so that $\left(\chi\left(x^{-1}, \gamma(x)\right)\right)(y) \leq \gamma(y)$ for every $y \in G$. This means that $\chi\left(x^{-1}, \gamma(x)\right) \subseteq \gamma$. By $a \leq \gamma(x), \chi\left(x^{-1}, a\right) \subseteq \gamma$.

Conversely, suppose that $\gamma$ satisfies the both conditions (FSG3), (FSG4). Let $x, y$ be the arbitrary elements of $G$. If, for example, $x \notin \operatorname{Supp}(\gamma)$, then $\gamma(x)=0$. It follows that $\gamma(x) \wedge \gamma(y)=0$, and hence $\gamma(x y) \geq 0=\gamma(x) \wedge \gamma(y)$. Therefore assume that $x, y \in \operatorname{Supp}(\gamma)$. Then $($ FSG3) shows that $\chi(x, \gamma(x)) \odot \chi(y, \gamma(y)) \subseteq \gamma$. By Proposition 1 of paper [4]

$$
\gamma(x) \wedge \gamma(y)=(\chi(x, \gamma(x)) \odot \chi(y, \gamma(y)))(x y) \leq \gamma(x y)
$$

thus we obtain $\gamma(x) \wedge \gamma(y) \leq \gamma(x y)$, and $\gamma$ satisfies (FSG1).

Let $x \in G$. Since $\chi\left(x^{-1}, \gamma(x)\right) \subseteq \gamma,\left(\chi\left(x^{-1}, \gamma(x)\right)\right)(y) \leq \gamma(y)$ for every $y \in G$. In particular,

$$
\left(\chi\left(x^{-1}, \gamma(x)\right)\right)\left(x^{-1}\right)=\gamma(x) \leq \gamma\left(x^{-1}\right),
$$

so that $\gamma$ satisfies (FSG2).

Proposition 2. Let $G$ be a group and $\gamma$ be a fuzzy subgroup on $G$. Then the center $\mathfrak{z}(\gamma)$ is a fuzzy subgroup of $\gamma$. 
Proof. Suppose that $\chi\left(z_{1}, a_{1}\right), \chi\left(z_{2}, a_{2}\right) \subseteq \mathfrak{z}(\gamma)$. Then

$\chi\left(z_{1}, a_{1}\right) \odot \chi(y, \gamma(y))=\chi(y, \gamma(y)) \odot \chi\left(z_{1}, a_{1}\right), \chi\left(z_{2}, a_{2}\right) \odot \chi(y, \gamma(y))=\chi(y, \gamma(y)) \odot \chi\left(z_{2}, a_{2}\right)$

for every element $y \in \operatorname{Supp}(\gamma)$. By proposition 1 of paper [4] $\chi\left(z_{1}, a_{1}\right) \odot \chi\left(z_{2}, a_{2}\right)=$ $\chi\left(z_{1} z_{2}, a_{1} \wedge a_{2}\right)$. We have now

$$
\begin{aligned}
\chi\left(z_{1} z_{2}, a_{1} \wedge a_{2}\right) \odot \chi(y, \gamma(y)) & =\left(\chi\left(z_{1}, a_{1}\right) \odot \chi\left(z_{2}, a_{2}\right)\right) \odot \chi(y, \gamma(y))= \\
\chi\left(z_{1}, a_{1}\right) \odot\left(\chi\left(z_{2}, a_{2}\right) \odot \chi(y, \gamma(y))\right) & =\chi\left(z_{1}, a_{1}\right) \odot\left(\chi(y, \gamma(y)) \odot \chi\left(z_{2}, a_{2}\right)\right)= \\
\left(\chi\left(z_{1}, a_{1}\right) \odot \chi(y, \gamma(y))\right) \odot \chi\left(z_{2}, a_{2}\right) & =\left(\chi(y, \gamma(y)) \odot \chi\left(z_{1}, a_{1}\right)\right) \odot \chi\left(z_{2}, a_{2}\right)= \\
\chi(y, \gamma(y)) \odot\left(\chi\left(z_{1}, a_{1}\right) \odot \chi\left(z_{2}, a_{2}\right)\right) & =\chi(y, \gamma(y)) \odot \chi\left(z_{1} z_{2}, a_{1} \wedge a_{2}\right) .
\end{aligned}
$$

Hence $\chi\left(z_{1} z_{2}, a_{1} \wedge a_{2}\right) \subseteq \mathfrak{z}(\gamma)$. In other words, $\chi\left(z_{1}, a_{1}\right), \chi\left(z_{2}, a_{2}\right) \subseteq \mathfrak{z}(\gamma)$ implies that $\chi\left(z_{1}, a_{1}\right) \odot \chi\left(z_{2}, a_{2}\right) \subseteq \mathfrak{z}(\gamma)$, so $\mathfrak{z}(\gamma)$ satisfies (FSG3).

Suppose now that $\chi(z, a) \subseteq \mathfrak{z}(\gamma)$. By Corollary $1 \operatorname{Supp}(\mathfrak{z}(\gamma))=\zeta(\operatorname{Supp}(\gamma))$. Since $\zeta(\operatorname{Supp}(\gamma))$ is a $\operatorname{subgroup}$ of $\operatorname{Supp}(\gamma), z^{-1} \in \zeta(\operatorname{Supp}(\gamma))$. It follows that $z^{-1} y=y z^{-1}$. Lemma 1 shows that $\chi\left(z^{-1}, a\right) \odot \chi(y, \gamma(y))=\chi(y, \gamma(y)) \odot \chi\left(z^{-1}, a\right)$, so that $\chi\left(z^{-1}, a\right) \subseteq$ $\mathfrak{z}(\gamma)$. Thus $\mathfrak{z}(\gamma)$ satisfies (FSG4). Proposition 1 proves that $\mathfrak{z}(\gamma)$ is a fuzzy subgroup on $\mathrm{G}$.

Corollary 4. Let $G$ be a group and $\gamma$ be a fuzzy subgroup on $G$. Then the center $\mathfrak{z}(\gamma)$ is a normal fuzzy subgroup of $\gamma$.

Proof. Indeed, $\mathfrak{z}(\gamma)$ is a subgroup of $\gamma$ by Proposition 2. An application of Corollary 3 and Proposition 4 of paper [4] yields that $\mathfrak{z}(\gamma)$ is a normal fuzzy subgroup of $\gamma$.

In the paper [4] has been constructed an upper central series of a fuzzy group $\gamma$. Further we will need the construction of the second hypercenter, therefore we recall it.

Put $\mathfrak{z}_{1}(\gamma)=\mathfrak{z}(\gamma)$. Without loss of generality we may assume that $\operatorname{Supp}(\gamma)=G$. Then Corollary 1 shows that $\operatorname{Supp}(\mathfrak{z}(\gamma))=\zeta(G)=Z_{1}$. Consider a factor-group $G / Z_{1}$ and let $\phi: G \rightarrow G / Z_{1}$ be a natural epimorphism. We can extend $\phi$ to the mapping $\Phi$ from the set $\mathcal{F}(G)$ of all fuzzy groups on $G$ to the set $\mathcal{F}\left(G / Z_{1}\right)$ of all fuzzy groups on $G / Z_{1}$ (see, for example, [[5], 1.1.11]). Define the function $\gamma^{\curlywedge}: G / Z_{1} \rightarrow[0,1]$ by the following rule: $\gamma^{\curlywedge}\left(g Z_{1}\right)=\bigvee_{z \in \zeta(G)} \gamma(g z)$. In other words, $\gamma^{\curlywedge}=\Phi(\gamma)$. Then $\gamma^{\curlywedge}$ is a fuzzy group on $G / Z_{1}$ (see, for example, [[5], Theorem 1.3.13]). Put $\mathfrak{z}\left(\gamma^{\curlywedge}\right)=\lambda$ and define the function $\mathfrak{z}_{2}(\gamma): G \rightarrow[0,1]$ in a following way. Consider a preimage $\lambda_{\curlyvee}$ of $\lambda$, that is the function $\lambda_{\curlyvee}: G \rightarrow[0,1]$, defined by the rule: $\lambda_{\curlyvee}(x)=\lambda\left(x Z_{1}\right), x \in G$. Put now $\mathfrak{z}_{2}(\gamma)=\lambda_{\curlyvee} \cap \gamma$.

Theorem 1. Let $G$ be a group and $\gamma$ be a fuzzy subgroup on $G$. Then $\mathfrak{z}_{2}(\gamma)=$ $\left.\zeta_{2}(\operatorname{Supp}(\gamma))\right|^{\gamma}$.

Proof. Without loss of generality we can suppose that $\operatorname{Supp}(\gamma)=G$. Let $g \in G, u \in$ $\operatorname{Supp}\left(\mathfrak{z}_{2}(\gamma)\right)$, and consider now a commutator $\left[\chi\left(u, \mathfrak{z}_{2}(\gamma)(u)\right), \chi(g, \gamma(g))\right]$. By Lemma 
2 of paper [4] $\left[\chi\left(u, \mathfrak{z}_{2}(\gamma)(u)\right), \chi(g, \gamma(g))\right]=\chi\left([u, g], \mathfrak{z}_{2}(\gamma)(u) \wedge \gamma(g)\right)$. By Lemma 3 of paper [4]

$$
\Phi\left(\chi\left([u, g], \mathfrak{z}_{2}(\gamma)(u) \wedge \gamma(g)\right)\right)=\chi\left([u, g] Z_{1}, \mathfrak{z}_{2}(\gamma)(u) \wedge \gamma(g)\right) .
$$

Using again Lemma 2 of paper [4], we obtain

$$
\chi\left([u, g] Z_{1}, \mathfrak{z}_{2}(\gamma)(u) \wedge \gamma(g)\right)=\left[\chi\left(u Z_{1}, \mathfrak{z}_{2}(\gamma)(u)\right), \chi\left(g Z_{1}, \gamma(g)\right)\right] .
$$

Since $\gamma^{\curlywedge}\left(g Z_{1}\right)=\bigvee_{z \in \zeta(G)} \gamma(g z)$ and $e \in Z_{1}$, then $\gamma^{\curlywedge}\left(g Z_{1}\right) \geq \gamma(g)$, so that $\chi\left(g Z_{1}, \gamma(g)\right) \subseteq \chi\left(g Z_{1}, \gamma^{\curlywedge}\left(g Z_{1}\right)\right)$. An inclusion $\Phi\left(\mathfrak{z}_{2}(\gamma)\right) \subseteq \lambda$ shows that $\left[\chi\left(u Z_{1}, \mathfrak{z}_{2}(\gamma)(u)\right), \chi\left(g Z_{1}, \gamma(g)\right)\right] \subseteq\left[\lambda, \gamma^{\curlywedge}\right]$. The choice of $\lambda$ together with Corollary 4 of paper [4] shows that $\left[\lambda, \gamma^{\curlywedge}\right] \preccurlyeq \chi\left(e Z_{1}, \gamma^{\curlywedge}\left(e Z_{1}\right)\right)$. In particular, $\chi\left([u, g] Z_{1}, \mathfrak{z}_{2}(\gamma)(u) \wedge\right.$ $\gamma(g)) \subseteq \chi\left(e Z_{1}, \gamma^{\curlywedge}\left(e Z_{1}\right)\right)$, which follows that $Z_{1}=[u, g] Z_{1}=\left[u Z_{1}, g Z_{1}\right]$. In turn, it follows that $u \in \zeta_{2}(G)$, the second hypercenter of $\mathrm{G}$. This inclusion implies that $u Z_{1} \in \zeta\left(G / Z_{1}\right)$. By Corollary $1 \lambda\left(u Z_{1}\right)=\gamma^{\curlywedge}\left(u Z_{1}\right)$. On the other hand, by the definition $\gamma^{\curlywedge}$ we have $\gamma^{\curlywedge}\left(u Z_{1}\right)=\bigvee_{z \in \zeta(G)} \gamma(u z)$. Since $e \in Z_{1}, \gamma^{\curlywedge}\left(u Z_{1}\right) \geq \gamma(u)$. An equation $\mathfrak{z}_{2}(\gamma)=\lambda_{\curlyvee} \cap \gamma$ shows that

$$
\mathfrak{z}_{2}(\gamma)(u)=\lambda_{\curlyvee}(u) \wedge \gamma(u)=\lambda\left(u Z_{1}\right) \wedge \gamma(u)=\gamma^{\curlywedge}\left(u Z_{1}\right) \wedge \gamma(u)=\gamma(u) .
$$

It follows that $\left.\mathfrak{z}_{2}(\gamma) \preccurlyeq \zeta_{2}(\operatorname{Supp}(\gamma))\right|^{\gamma}$.

Conversely, let $u \in \zeta_{2}(G)$. Then $u Z_{1} \in \zeta\left(G / Z_{1}\right)$. Since $G=\operatorname{Supp}(\gamma), \gamma(u) \neq 0$. Corollary 1 shows that a fuzzy point $\chi\left(u Z_{1}, \gamma^{\curlywedge}\left(u Z_{1}\right)\right)$ lies in the center $\lambda$ of $\gamma^{\curlywedge}$. It follows that $\lambda\left(u Z_{1}\right)=\gamma^{\curlywedge}\left(u Z_{1}\right)$. An equation $\gamma^{\curlywedge}\left(u Z_{1}\right)=\bigvee_{z \in \zeta(G)} \gamma(u z)$ shows that $\gamma^{\curlywedge}\left(y Z_{1}\right) \geq \gamma(y)$. We have now

$$
\mathfrak{z}_{2}(\gamma)(u)=\left(\lambda_{\curlyvee} \cap \gamma\right)(u)=\lambda_{\curlyvee}(u) \wedge \gamma(u)=\lambda\left(u Z_{1}\right) \wedge \gamma(u)=\gamma^{\curlywedge}\left(u Z_{1}\right) \wedge \gamma(u)=\gamma(u),
$$

which shows that $\chi(u, \gamma(u)) \subseteq \mathfrak{z}_{2}(\gamma)$, and hence that $\left.\zeta_{2}(\operatorname{Supp}(\gamma))\right|^{\gamma} \preccurlyeq \zeta_{2}(\gamma)$.

The construction of the second hypercentre is the starting point for a construction of the upper central series of a fuzzy group $\gamma$. Using for this transfinite induction. Without loss of generality we may assume that $\operatorname{Supp}(\gamma)=G$. We have already constructed the terms $\mathfrak{z}_{1}(\gamma)=\mathfrak{z}(\gamma)$ and $\mathfrak{z}_{2}(\gamma)$. Suppose that we have already constructed the terms $\mathfrak{z}_{\beta}(\gamma)$ for all ordinals $\beta<\alpha$. If $\alpha$ is a limit ordinal, then we put $\mathfrak{z}_{\alpha}(\gamma)=\cup_{\beta<\alpha} \mathfrak{z} \beta(\gamma)$. Suppose now that $\alpha$ is a not limit ordinal, that is $\alpha-1=\eta$ exists. Let $K=\zeta_{\eta}(G)$, the $\eta^{\text {th }}$ term of an upper central series of $G$. Consider a factor-group $G / K$ and let $\phi: G \rightarrow G / K$ be a natural epimorphism. We can extend $\phi$ to the mapping $\Phi$ from the set $\mathcal{F}(G)$ of all fuzzy groups on $G$ to the set $\mathcal{F}(G / K)$ of all fuzzy groups on $G / K$ (see, for example, [[5], 1.1.11]). Define the function $\gamma^{\curlywedge}: G / K \rightarrow[0,1]$ by the following rule: $\gamma^{\curlywedge}(g K)=\bigvee_{z \in K} \gamma(g z)$. In other words, $\gamma^{\curlywedge}=\Phi(\gamma)$. Then $\gamma^{\curlywedge}$ is a fuzzy group on $G / K$ (see, for example, [[5], Theorem 1.3.13]). Put $\mathfrak{z}\left(\gamma^{\curlywedge}\right)=\lambda$ and define the function $\mathfrak{z}_{\alpha}(\gamma): G \rightarrow[0,1]$ in a following way. Consider a preimage $\lambda_{\curlyvee}$ of $\lambda$, that is the function $\lambda_{\curlyvee}: G \rightarrow[0,1]$, defined by the rule: $\lambda_{\curlyvee}(x)=\lambda(x K), x \in G$. Put now $\mathfrak{z}_{\alpha}(\gamma)=\lambda_{\curlyvee} \cap \gamma$.

Thus, for every ordinal $\alpha$ we constructed the $\alpha^{\text {th }}$ term $\mathfrak{z}_{\alpha}(\gamma)$ of an upper central series of $\gamma$. The building of an upper central series of $\gamma$ come to an end on some ordinal 
$\sigma$. In other words, this means that if $L=\zeta_{\sigma}(G)$ and $\gamma^{\curlywedge}(g L)=\bigvee_{z \in L} \gamma(g z)$, then the center of $\gamma^{\curlywedge}$ is $\chi\left(e, \gamma^{\curlywedge}(e)\right)$. Then $\mathfrak{z}_{\sigma}(\gamma)$ is called the upper hypercenter of $\gamma$ and will denoted by $\mathfrak{z}_{\infty}(\gamma)$.

Repeating almost word for word the arguments from the proof of Theorem 1 we prove the following result.

Theorem 2. Let $G$ be a group and $\gamma$ be a fuzzy subgroup on $G$. Let

$$
\chi(e, \gamma(e))=\mathfrak{z}_{0}(\gamma) \preccurlyeq \mathfrak{z}_{1}(\gamma) \preccurlyeq \ldots \preccurlyeq \mathfrak{z}_{\beta}(\gamma) \preccurlyeq \mathfrak{z}_{\beta+1}(\gamma) \preccurlyeq \ldots \preccurlyeq \mathfrak{z}_{\sigma}(\gamma)
$$

be the upper central series of $\gamma$. Then $\mathfrak{z}_{\beta}(\gamma)=\left.\zeta_{\beta}(\operatorname{Supp}(\gamma))\right|^{\gamma}$ for every ordinal $\beta$.

Corollary 5. [[4], Theorem 1] Let $G$ be a group, $\gamma$ be a fuzzy subgroup on $G$. Then $\gamma$ is hypercentral if and only if $\operatorname{Supp}(\gamma)$ is hypercentral.

\section{References}

1. Asaad M., Abou-Zaid S. Fuzzy subgroups of nilpotent groups. // Fuzzy Sets and Systems, 1993. - N. 24. - P. 321-323.

2. Gupta K. C., Sarma B. K. Nilpotent fuzzy groups. // Fuzzy Sets and Systems, 1999. N. 101. - P. 167-176.

3. Kim J. G. Commutative fuzzy sets and nilpotent fuzzy groups. // Information Sciences, 1995. - N. 83. - P. 161-174.

4. Kurdachenko L. A., Grin K. O., Turbay N. A. On hypercentral fuzzy groups. // Algebra and Discrete Mathematics, 2012. - Vol. 13, N. 1. - P. 92-106.

5. Mordeson J. N., Bhutani K. R., Rosenfeld A. Fuzzy Group Theory. - Berlin: Springer, 2005. 